\title{
Scale-Dependent Inhibition Drives Regular Tussock Spacing in a Freshwater Marsh
}

\author{
Johan van de Koppel ${ }^{1, *}$ and Caitlin Mullan Crain ${ }^{2, \dagger}$
}

1. Spatial Ecology Department, Centre for Estuarine and Marine Ecology, Netherlands Institute of Ecology (NIOO-KNAW), P.O. Box 140, NL-4400 AC Yerseke, The Netherlands;

2. Department of Ecology and Evolutionary Biology, Box G-W, Brown University, Providence, Rhode Island 02912

Submitted April 5, 2006; Accepted June 28, 2006; Electronically published October 13, 2006

Online enhancements: videos, Matlab code.

ABSTRACT: Regular spatial patterning is common in nature, and various mechanisms of self-organization have been proposed to explain regular patterning. We report on regular spatial patterning in Carex stricta in a freshwater wetland and investigate the applicability of theoretical models that explain regular patterning based on inhibition, facilitation, or interaction between the two. Spectral analysis of aerial photographs revealed that tussocks were regularly spaced at an average distance of $60 \mathrm{~cm}$. Photosynthetically active radiation varied significantly with distance from the tussock and was lowest at intermediate distance from the tussock center $(15-40 \mathrm{~cm})$. Using transplants to assay growth conditions, we found that $C$. stricta grew well in all distance classes with and without natural C. stricta biomass, except at intermediate distances when buried in C. stricta wrack. Our experimental results reveal that $C$. stricta inhibits its growth in a scale-dependent manner: inhibition was found to peak at intermediate distance from the tussock. We compared three alternative models to examine potential mechanisms driving regularity and found that, similar to our experimental results, scale-dependent inhibition provides the best explanation for the observed regular tussock spacing. Our study underlines the importance of scale-dependent feedback in the formation of regular spatial patterning in ecosystems.

Keywords: self-organization, facilitation, competition, regular patterns, tussock formation, scale-dependent interactions.

The occurrence of regular spatial patterning in ecosystems has been a dominant topic in the study of ecosystem com-

* E-mail: j.vandekoppel@nioo.knaw.nl.

† E-mail: caitlin_crain@brown.edu.

Am. Nat. 2006. Vol. 168, pp. E136-E147. () 2006 by The University of Chicago. 0003-0147/2006/16805-41743\$15.00. All rights reserved. plexity. Early studies, focusing on regular spacing of individual plants or trees, emphasized local inhibition due to competition for resources such as light in explaining regular spacing of individuals (Ford 1975; Kenkel 1988; Nisbet et al. 1997). A recent body of theory proposes scaledependent interaction between facilitation and inhibition among organisms to explain the formation of regular patterns in ecosystems. For example, regular spatial patterns observed in various ecosystems such as arid bushlands (Klausmeier 1999; Couteron and Lejeune 2001; Von Hardenberg et al. 2001; Rietkerk et al. 2004b), peatlands (Rietkerk et al. 2004a), and mussel beds (van de Koppel et al. 2005) have been theoretically linked to the interaction between competition and facilitation. The mechanism driving regular patterning in scale-dependence theory is qualitatively similar to the activator-inhibitor principle that was first proposed by Turing in the early 1950s (Turing 1952) for chemical systems. Turing's principle explains regular patterning by assuming that a species (either an organism or a catalyzing enzyme) promotes its own growth at small scales but inhibits its growth at larger spatial scales. While this principle has been proposed to apply to a wide range of patterned ecosystems (Rietkerk et al. 2004b), there is a conspicuous lack of experimental evidence for the presence of scale dependence in any of these systems.

In this article, we present an experimental investigation of regular spatial patterning in Carex stricta and use this ecosystem as a model to investigate alternate theoretical mechanisms that drive regular spatial patterning in general. Carex stricta, the tussock sedge, is a species with widespread distribution in freshwater marshes of North America. Tussock formation in wetland plants is a fairly common occurrence and appears to be a stress avoidance strategy for plants that are able to escape waterlogging and associated low oxygen levels and soil toxicity by elevating their rooting substrate. While tussock formation can be induced in some species (e.g., Triglochin maritimum; Fogel et al. 2004), in C. stricta the habit appears fixed because C. stricta grows almost exclusively in large tussocks (Costello 1936; Crain and Bertness 2005). Tussock formation is the result of concentrated rooting by 
plants that position their roots above or just in the top soil layer. This stimulates sediment capture and retention, leading to the formation of a small mound on which plant growth is focused. In some wetland systems, mounding has been shown to lead to more benign environmental conditions that promote plant growth (Figueroa et al. 2003; Fogel et al. 2004; Ervin 2005). In tidal freshwater marshes, C. stricta appears less dependent on mounding for evading waterlogging, at least in developed marshes, and instead benefits from growing on top of mounds that provide refuge from small mammalian herbivores that forage in intertussock areas (Crain and Bertness 2005). However, C. stricta also inhibits its own recruitment and growth by producing large amounts of standing dead plant material that falls radially from the tussock and accumulates as wrack (fig. 1). This wrack material inhibits the growth of C. stricta and other species by reducing light levels and providing a physical barrier to plant emergence (Crain and Bertness 2005). The way in which self-facilitation or inhibition, and their dependence on spatial scale, in fact drive regular spatial patterning of vegetation in this system remains to be experimentally tested.

We studied the spatial distribution of $C$. stricta tussocks and investigated potential explanatory mechanisms for the observed vegetative patterns. We used spectral analysis of short-range aerial photographs to establish whether tussock vegetation indeed exhibits a regular spatial patterning. We investigated whether $C$. stricta affects light availability at the soil surface in a scale-dependent matter. Using a manipulative transplant experiment, we tested the hypothesis that growth of $C$. stricta transplants is strongly limited at intermediate distance from the tussock, rather than at tussock centers, and that this reduction is related to the presence of wrack produced by conspecifics. Finally, we compared our experimentally derived mechanisms with other hypothesized mechanisms of regular patterning by analyzing three alternative spatially explicit models of

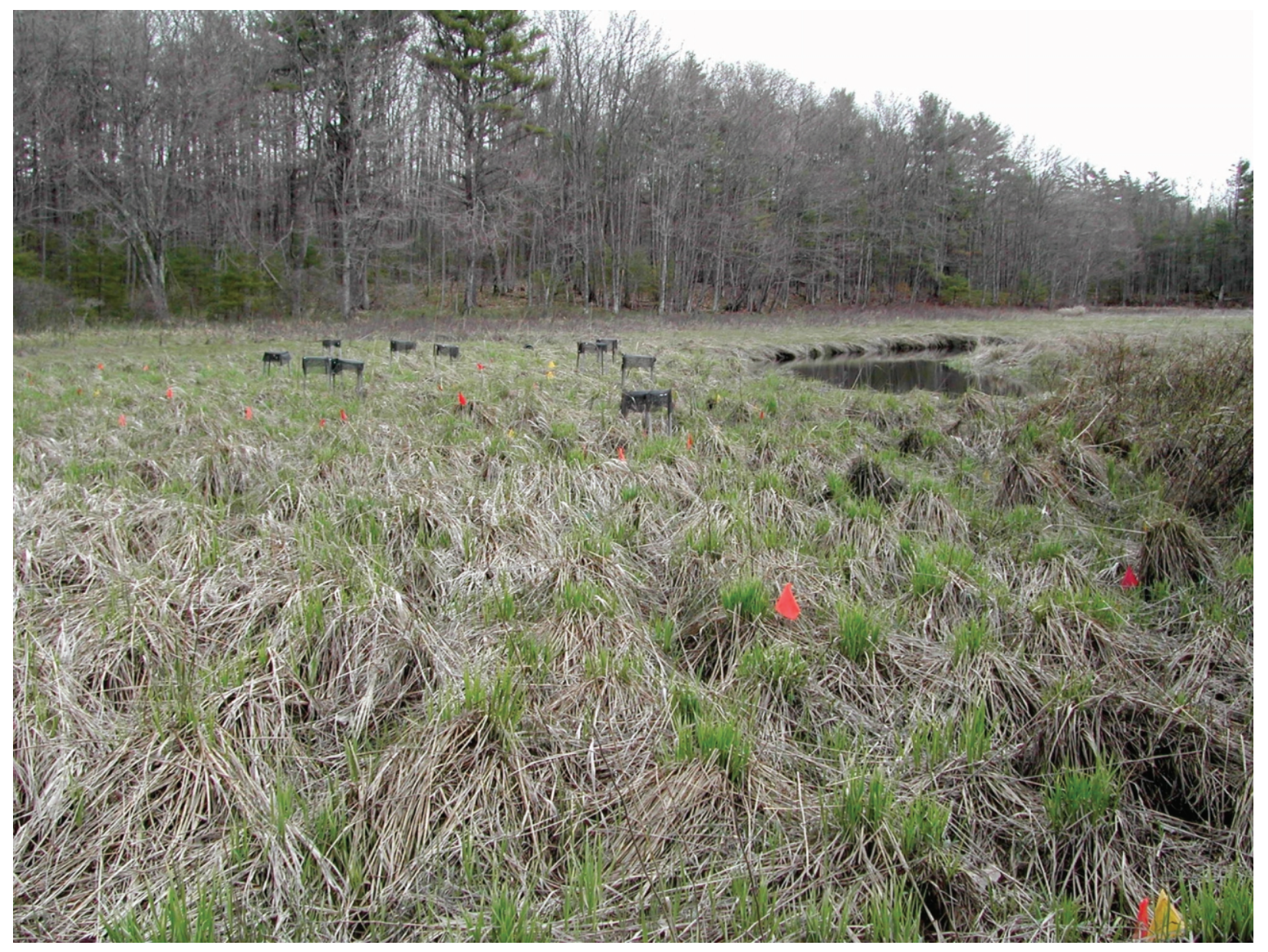

Figure 1: Picture of a Carex stricta tussock marsh in early spring. The flags show the locations of the experimental replicates. 
vegetation patterning. The model analysis enables us to investigate which mechanism is the most likely explanation for driving regular spatial patterning in this system. We discuss the proposed explanation in light of scaledependence theory.

\section{Methods}

Field Site

We studied the spatial distribution of Carex stricta tussocks in a tidal freshwater marsh that receives semidiurnal tidal inundation from the Branch Brook fork of the Little River in the Wells National Estuarine Research Reserve/Rachel Carson National Wildlife Refuge in southern Maine. Carex stricta, the tussock sedge, dominates the low intertidal zone in the marsh, particularly on the inside banks of oxbow turns of the Little River. In this zone, C. stricta grows almost exclusively in tussocks about $25 \mathrm{~cm}$ high and 40 $\mathrm{cm}$ in diameter. Carex stricta produces large amounts of litter, which is dropped in a circular fashion around the tussock and subsequently accumulates as wrack (fig. 1). The intertussock spaces average $20 \mathrm{~cm}$ across and are unvegetated due to the effects of adverse edaphic conditions, wrack burial, and grazing by small rodents (Crain and Bertness 2005).

\section{Spatial Analysis of Vegetation}

Visual observation strongly suggested that the tussocks were regularly spaced. This implies that the position of a tussock depends on the presence of neighboring tussocks, and hence that ecological interactions are potentially important determinants of the spatial structure of C. stricta vegetation. We studied the spatial distribution of C. stricta tussocks using aerial photographs to establish statistically that the observed tussocks were regularly distributed rather than randomly spaced and to provide a rough estimate of the average intertussock distance. Digital images were taken on May 12, 2004, using a Nikon Coolpix 5000 with an additional wide-angle lens (Nikon WC-E68) mounted on a 6-m fishing rod; the images were taken using the camera's timer. Replicate $5 \times 5$-m-square sections were obtained from the middle of the original digital images to minimize fisheye distortion. When pictures were taken in early spring, young shoots were just emerging from the wrack, clearly marking the position of the tussocks on the images. We extracted the green coloration from the images by applying the following equation to the image pixels: $E=G-(R+B) / 2$, where $G, R$, and $B$ were the green, red, and blue bands in the image, respectively. Where green shoots emerge from tussock tops, $E$ is positive, so positive values were extracted and negative values were set to 0 . Gradients in lighting that were caused by sunlight reflection were removed from the images by using a sliding-block filter that rescales the image relative to the maximum $E$ value within each block. Images were resized to $50 \times 50$ pixels to exclude variance at scales below $10 \mathrm{~cm}$, which is irrelevant to the current study.

We tested for regular spacing of the tussocks by applying a two-dimensional spectral analysis (Renshaw and Ford 1983; Couteron and Lejeune 2001) to the extracted images. Spectral analysis provides a measure of the amount of variance within the image, referred to as $I_{l, d}$, that is explained by a simple cosine with a specific wavelength $l$ and direction $d$. To investigate the dependence of $I$ on wavelength, we plotted the radial spectrum of $I$, which reveals the dominant number of waves that fit within the analyzed image, irrespective of direction. A regular pattern within the image will be reflected by a peak in $I$ at a certain wave number. Detailed treatments of the method can be found in articles by Renshaw and Ford (1983), Mugglestone and Renshaw (1998), and Couteron and Lejeune (2001). Although our images suffer from slight fish-eye distortion caused by the wide-angle lens, this distortion obscures only regular spacing, and hence our analysis provides a conservative estimate of regularity.

\section{Transplant Experiment}

In order to examine scale-dependent positive and negative impacts of C. stricta on growth of conspecifics, we tested plant performance at varying distances from the tussock and in the presence and absence of naturally occurring aboveground standing crop, which was a mix of live shoots and wrack. This design enabled us to test whether plant growth patterns are driven by environmental variables or interactions with aboveground plant material and how these interactions change in intensity or direction with distance from the tussock. Performance of transplanted units of C. stricta was reanalyzed from a previous experiment designed to examine how ecosystem engineering by C. stricta affected the vegetative community through tussock manipulation and transplants of several common species (Crain and Bertness 2005). In our study, we reanalyzed a subset of the experimental results to specifically address how plants performed with respect to distance from the tussock center, and we additionally excluded aboveground C. stricta standing crop to examine whether the strength or direction of plant interactions (aboveground competition or facilitation) varied with distance.

Units of C. stricta were transplanted as phytometers to determine how plant performance varied across experimental treatments. In early April 2002, single emerging shoots of C. stricta and the associated rhizome were cut 


\section{Original image}
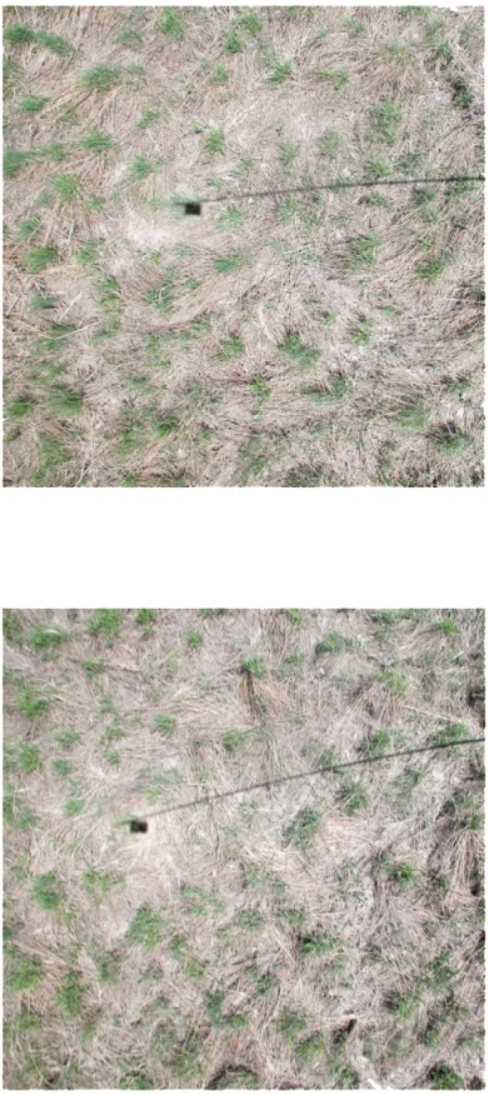

Size of area: appr. $5 \times 5$ meter

\section{Spectral analysis}
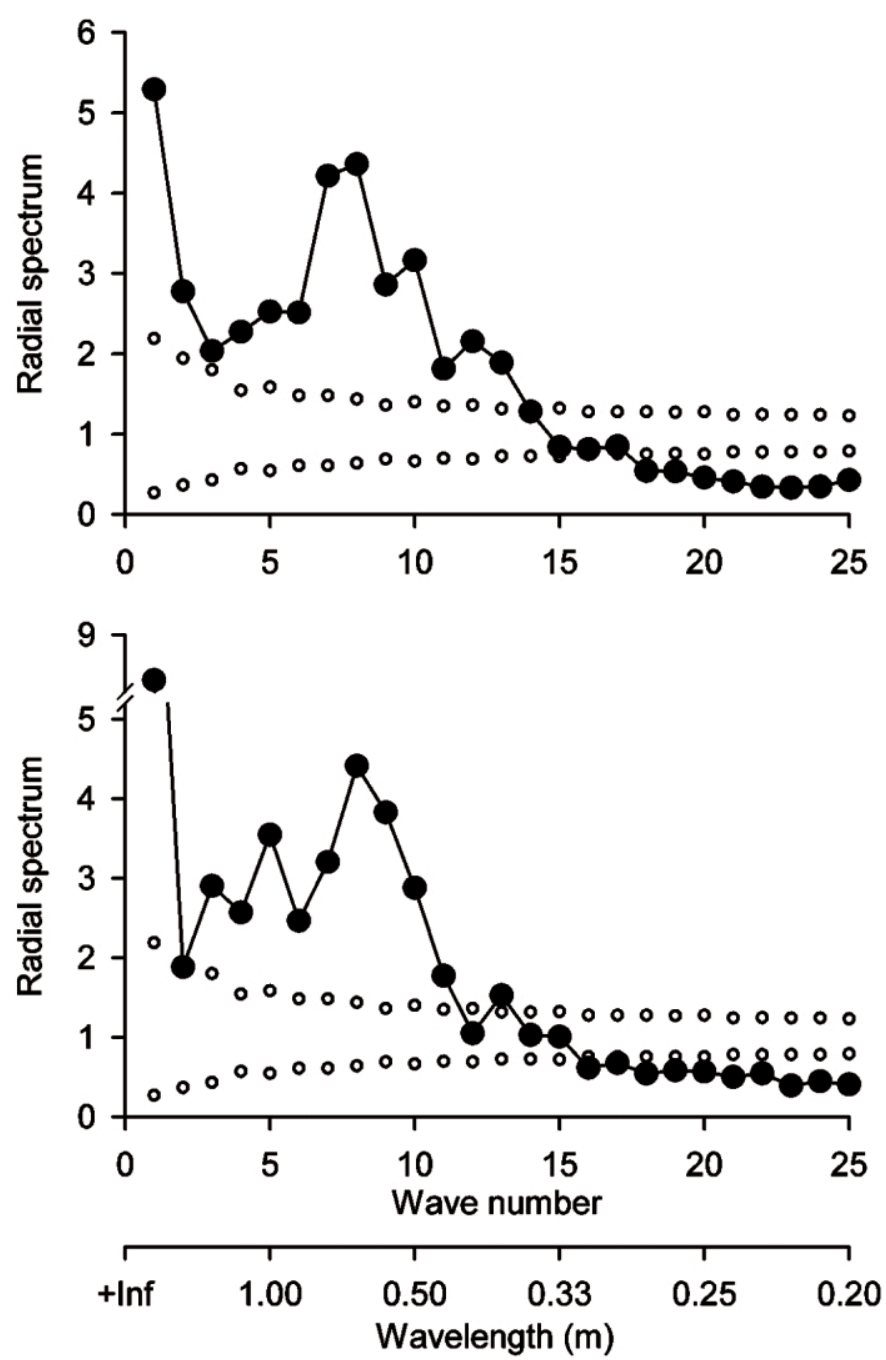

Figure 2: Spectral analysis of two images of the Carex stricta tussock vegetation. The two graphs represent the amount of variance explained by a cosine with a wave number (number of waves within the image), as specified by the $X$-axis. Dotted lines represents the $5 \%$ bilateral confidence interval for radial spectra ( $\chi^{2}$ distribution). Note that the dark line and square on the original images, which are the shadows of the fishing pole and the camera, were removed from the image by our green extraction algorithm.

from the local marsh and transplanted into experimental treatments. To test the effect of distance from tussock center, we had three distance classes: on top of a tussock (0 $\mathrm{cm})$, directly next to a tussock $(15 \mathrm{~cm})$, and far from a tussock $(75 \mathrm{~cm})$. Because the $75-\mathrm{cm}$ distance class coincided with other tussocks, for these treatments we effectively recreated a nontussocked marsh by lowering tussocks to be flush with the background soil elevation (see Crain and Bertness 2005). This effectively created a "far" distance class without complications of height or lack of vegetation, best mimicking a marsh in the absence of the tussocks themselves. In each of these distance classes, we had one unmanipulated treatment, reflecting the natural environmental conditions at that distance, and a second treatment removing the aboveground influence of $C$. stricta. The resultant treatments were (1) raised tussock with and without standing crop of C. stricta, (2) intertussock mud with and without $C$. stricta wrack deposition (no live plant parts are found here), and (3) lowered tussocks with and without $C$. stricta standing crop at 0,15 , 


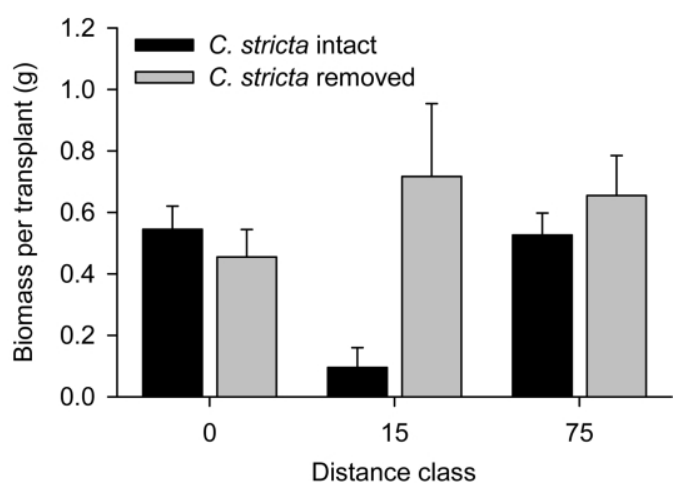

Figure 3: Performance of Carex stricta transplants at varying distance from tussock centers with and without aboveground standing crop (including wrack). Carex stricta removals vary depending on distance class; at 0 and $75 \mathrm{~cm}$, aboveground shoots and wrack of C. stricta were removed, while at $15 \mathrm{~cm}$, only C. stricta wrack was removed, as live plants did not occur here.

and $75 \mathrm{~cm}$, respectively. Each treatment was conducted independently and replicated 10 times.

Transplant performance was followed for one growing season, and in August 2002, aboveground biomass was harvested, dried, and weighed. Biomass data were $\log (x+1)$ transformed to improve homogeneity of variance and analyzed in a two-way ANOVA testing the effects of distance, C. stricta biomass, and their interaction on plant performance. Data were analyzed using JMP statistical software, and post hoc comparisons were performed using JMP's "least square means contrast" feature.

\section{Light Availability}

Based on previous work in the system, light reduction was identified as a primary mechanism driving spatial patterning of vegetation in tussock marshes (Crain and Bertness 2005). Carex stricta accumulates extensive amounts of standing dead, which eventually falls radially from the tussock and accumulates in intertussock areas, inhibiting further colonization via seedlings or even vegetative runners. To investigate how shading by wrack and conspecifics varies with distance from the center of individual tussocks, we analyzed the relationship between light availability at the soil surface and distance from the tussock center. We determined light intensity levels (photosynthetically active radiation $[\mathrm{PAR}]$ ) at the soil surface at 5 -cm intervals along 10 transects from a tussock into the intertussock space, using a different tussock for each transect. Transects began at the center of an elevated tussock at the soil surface and moved horizontally from the center at ground level, dropping to the elevation of the soil matrix. Transect angle was selected in such a way that the longest distance could be covered without running into another tussock. Light levels (PAR in $\mu \mathrm{mol} / \mathrm{m}^{2} / \mathrm{s}$ ) were measured with a LiCor line quantum sensor (which integrates light readings over 0.5 $\mathrm{m})$ aligned perpendicular to the transect on a cloudless day in May. We measured background light levels just before and after measuring the transects to compensate for changes in sunlight intensity. After logarithmic transformation of the light levels, we analyzed the relation between distance and light levels by fitting a general linear model with a polynomial fit.

\section{Empirical Results}

\section{Spatial Analysis of Vegetation}

The spectral analysis revealed clear regular spacing of the tussocks on the investigated images (two of which are presented in fig. 2), with a peak wave number of about 8. This concurs with a tussock distance of about $62.5 \mathrm{~cm}$ and is in accordance with the $61-\mathrm{cm}$ distance reported by Crain and Bertness (2005). The analysis revealed that some wave numbers lower than 3 also explain a significant part of the variance. This is likely due to differences in the size of the tussocks, the intensity of green on the image, and large-scale spatial trends on the pictures such as the depressed intensity of green coloring at the center of the image.

\section{Transplant Experiment}

Performance of Carex stricta transplants clearly showed that inhibition of growth by aboveground competition varied with distance from tussock centers (distance $\times$ competition interaction: $F=6.22$, $\mathrm{df}=1,49, p=.0042$; fig. $3)$. The treatment responsible for driving this variation

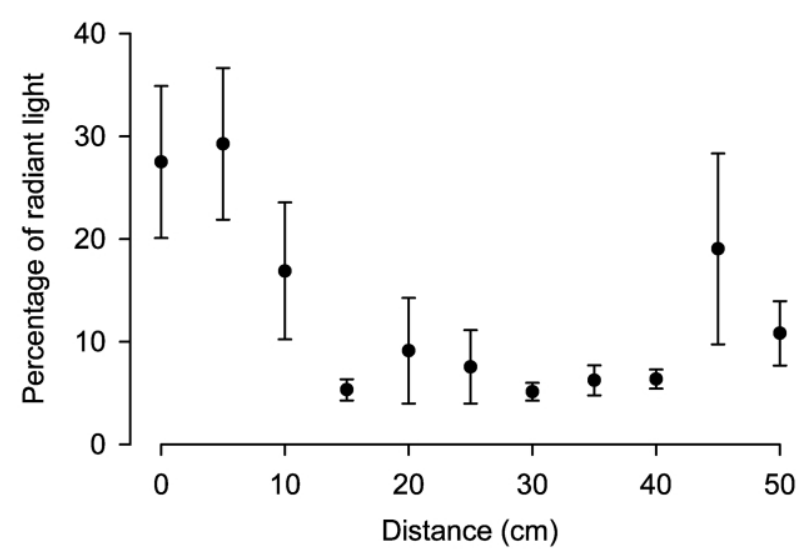

Figure 4: Light levels on top of the soil, expressed as percentage of radiant light, versus distance from tussock center. 
Model 1: Inhibition only

C. stricta biomass

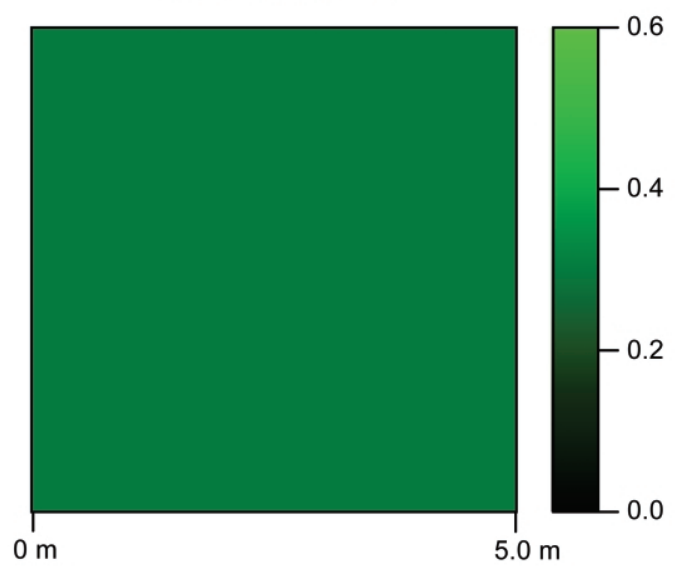

Wrack

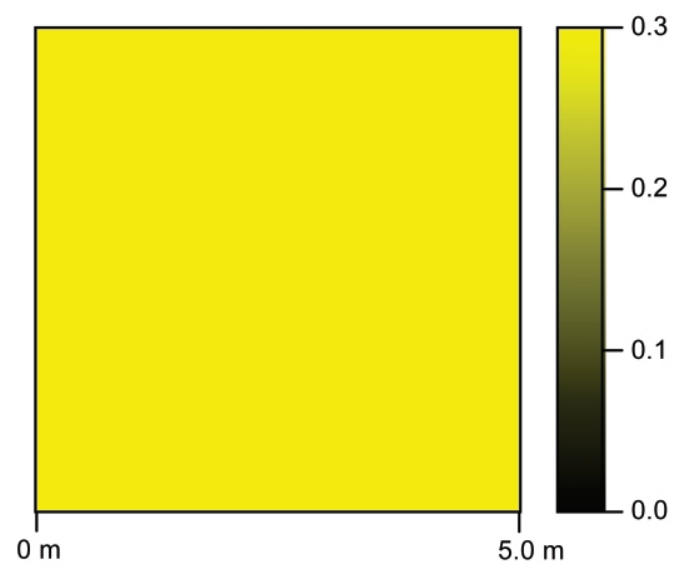

Model 2: Interaction between facilitation and inhibition

C. stricta biomass

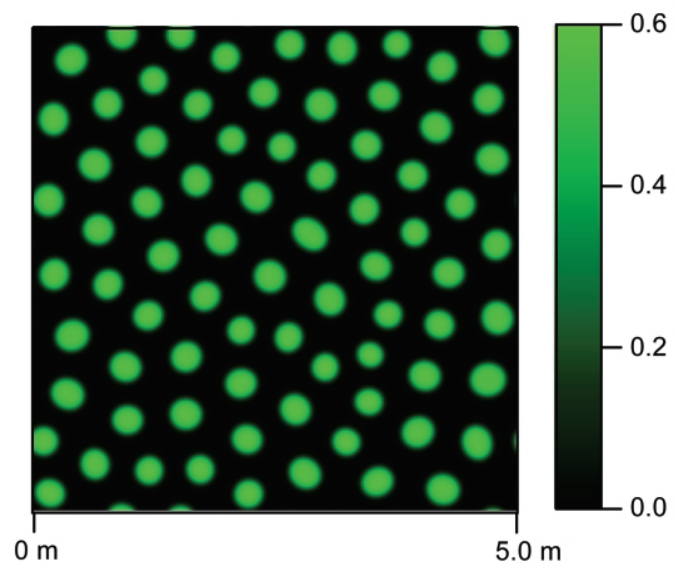

Wrack

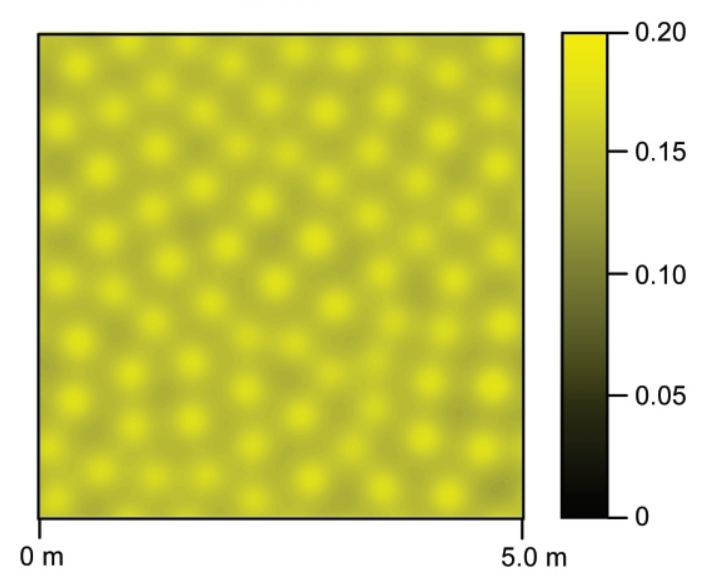

Model 3: Inhibition only at intermediate range

C. stricta biomass

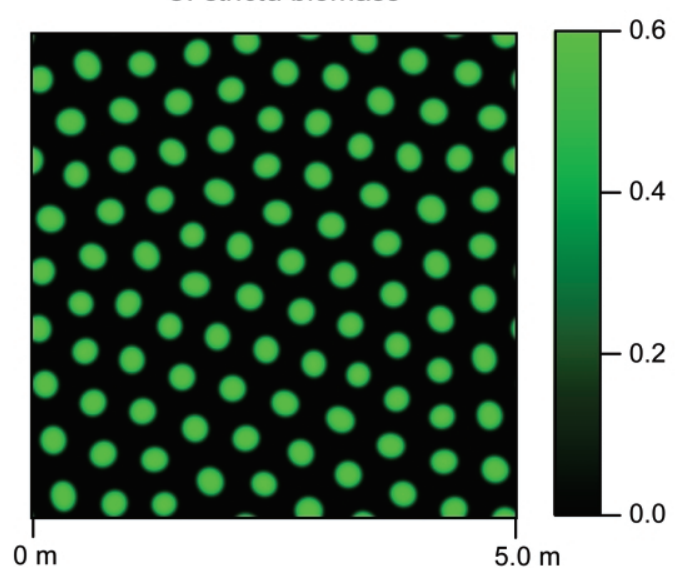

Wrack

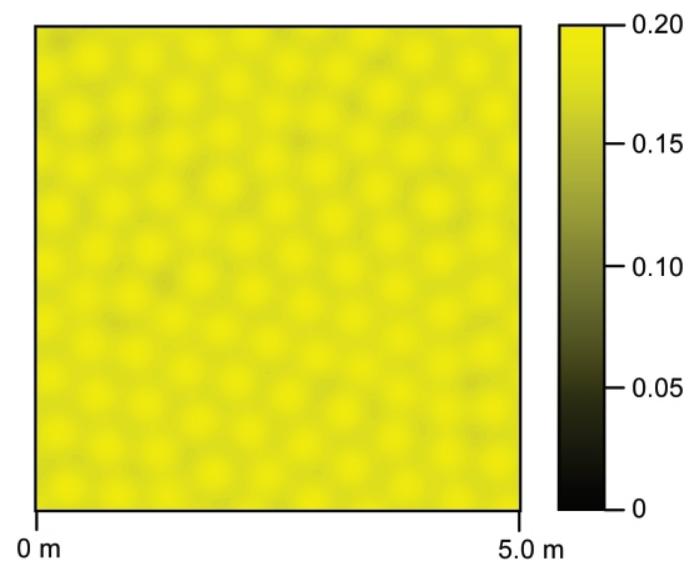


was the $15-\mathrm{cm}$ distance with $C$. stricta wrack intact, which varied significantly from all other treatments (least squares contrast: $F=25.75$, df $=1,44, p<.00001)$. This treatment is representative of natural intertussock spaces.

\section{Light Availability}

Base light measurements showed that light levels varied with distance from the tussocks (fig. 4). The highest light levels were observed on top of the tussocks, while light levels were strongly reduced underneath the wrack at intermediate distance, right next to the tussocks. Light levels decreased significantly with distance (linear regression of log-transformed data, effect of distance: $F=37.4$, df $=$ $1,107, p<.001)$ but increased again at the end of the transects (regression with polynomial fit on log-transformed data, effect of distance squared: $F=29.6$, df $=1,107$, $p<.001$ ), when past the wrack and beyond the sphere of tussock influence.

\section{Analyzing Alternative Scale-Dependent Feedback Models}

\section{Model Formulation}

When explaining the occurrence of regular spacing of individual plants or trees, ecological literature emphasizes the importance of competition limiting the success of conspecifics at short distance (Kenkel 1988; Ford and Sorrensen 1992). Recent theoretical studies on regular dotted vegetation patterns emphasize the importance of scaledependent interaction between positive and negative feedbacks (Couteron and Lejeune 2001; Lejeune et al. 2002; Rietkerk et al. 2004b). Our empirical study suggests a middle ground: although the direct positive effect of tussocks on their own survival through the development of root mounds might be limited (fig. 3; 0 vs. $75 \mathrm{~cm}$ ), tussocks elevate the plants above the wrack layer and effectively alleviate inhibition by the wrack material. Hence, inhibition in the tussock marsh appears to be scale dependent, peaking at intermediate distance.

To investigate which of these hypothesized mechanisms could, theoretically, best explain the observed spatial regularity of tussock vegetation, we analyzed and compared these alternative mechanisms, using three simple, spatially explicit models describing the growth of Carex stricta. These models follow the general structure

$$
\begin{aligned}
\frac{d P}{d t} & =P(1-P) F(P)-s P-I(P, W)+d_{\mathrm{P}} \Delta P, \\
\frac{d W}{d t} & =s P-b W+d_{\mathrm{W}} \Delta W .
\end{aligned}
$$

Here, $P$ is plant biomass, $W$ is wrack biomass, $F(P)$ is a function describing the positive effect of plant biomass on its own growth, $s$ is the specific rate of plant senescence, $I(P, W)$ is a function describing the inhibiting effect of wrack on plant growth as a function of plant and wrack biomass, $b$ is the decay rate of wrack, and $d_{\mathrm{p}}$ and $d_{\mathrm{w}}$ are diffusion constants describing lateral movement of plants and wrack. We adopted diffusion, being a linear function of the Laplacian operator (Holmes et al. 1994), as a crude approximation of lateral movement of both living material (e.g., through tillers) and wrack (falling from the plant or moved by the tides). Our model therefore likely underestimates the accumulation of wrack in the intertussock spaces. We adopted this simplifying approximation to be able to link our model to other pattern studies in literature and to focus on the alternative mechanisms describing facilitation or inhibition. A further yet critical assumption for pattern formation is that the rate of spatial spread of wrack surpasses that of plants, for example, via tillers. Finally, we used dispersive kernels as explained in Powell and Zimmerman (2004) to model diffusion of plants and wrack.

In the first model, we consider only negative interactions between plants through the production of wrack: $F(P)=1$ and $I(P, W)=i P W$, where $i$ is a constant translating wrack biomass to specific plant growth inhibition. The second model closely follows the pattern models found in the literature that assume scale-dependent positive and negative feedback of the vegetation on its own growth. In this model, $F(P)=P /\left(P+k_{2}\right)$ and $I(P, W)=i P W$, where $k_{2}$ is a self-facilitation constant describing the positive effect of vegetation on its own growth, which occurs in our system through the development of a root mound. The last model closely follows our own

Figure 5: Vegetation structure predicted by three models with different mechanistic descriptions of the interactions between Carex stricta conspecifics and wrack material. The first model includes only a direct inhibitory effect of wrack on C. stricta growth. The second model builds on the first model by including a direct facilitory effect of $C$. stricta on itself via mound formation. The third model expands on the first by including an alleviating effect of $C$. stricta biomass on wrack inhibition, labeled in the article as indirect facilitation. In the online edition, the results of simulations of models 1-3 are shown in three videos of the spatial development of vegetation. Note that the simulations in the videos lasted only 500 time steps, while the results in the figure were obtained after 2,000 time steps. This was done to limit the duration of the videos, since the spatial development after 500 time steps is very slow. As a consequence, regularity is less well developed in the videos. Parameter values for the simulations (see app. B): $b=0.1 ; d_{\mathrm{P}}=0.0001 ; d_{\mathrm{D}}=0.01 ; s=0.15$; model $1: i=1$; model $2: i=1, k_{2}=0.3 ;$ model $3: i=10 ; k_{3}=0.2$. 
empirical results and assumes that the positive effect of concentration of biomass acts through the lowering of inhibition by the wrack as a consequence of mound building. In this model, we assume that $F(P)=1$ and $I(P, W)=i P W k_{3} /\left(P+k_{3}\right)$, where $k_{3}$ is the level of plant biomass where inhibition is lowered by half. The models are analyzed by simulating the spatial development of the vegetation after random seeding on a spatial grid of $512 \times 512$ cells, reflecting an area of $5 \mathrm{~m} \times 5 \mathrm{~m}$. In appendix $B$, we provide additional information about the simulation methodology.

\section{Modeling Results}

No pattern formation is found for any parameter combination in the first model, where inhibition decreases with distance between plants (fig. 5); the model develops to a homogeneous stand of vegetation despite strong heterogeneity introduced by the initial seeding (see the fig. 5 legend in the online edition of the American Naturalist to link to videos of the simulations). This concurs with the theoretical literature on the development of spatial patterning (Turing 1952; Klausmeier 1999; Rietkerk et al. $2004 b$ ) but contrasts with the empirical literature on regular spacing of plants (Ford 1975; Nisbet et al. 1997). Dotted patterns do develop in the second (scale-dependent positive and negative feedback) and third (scale-dependent inhibition) models (fig. 5). In both models, tussocks develop following random seeding and develop a halo of wrack around them that prevents the growth of vegetation in the intertussock spaces (note that due to the diffusion approximation, wrack density is highest on top of the tussocks, while in nature, wrack densities are typically higher in the intertussock spaces). This result suggests that a scaledependent inhibitory effect of $C$. stricta on its own growth, in which inhibition is strongest at some distance from the tussock center, is key to explaining the formation of regular tussock spacing. Net inhibition is low on top of the tussock, either because the effect of the wrack is ameliorated on top of the tussock (model 3) or by a facilitative effect of the tussock on plant growth, for example, by improved edaphic conditions (model 2). Both mechanisms can explain regular spacing of clumps of vegetation. With the parameter settings on which figure 5 is based, models 2 and 3 predict clumps of vegetation. In both models, if inhibition of plant growth $i$ or senescence rate $s$ is lowered, the clumps merge to produce a labyrinth-type vegetation pattern, typical for Turing-type models of spatial patterns (not shown; see, e.g., Rietkerk et al. 2004b). We observed similar patterns in the field when tussocks lined up to create elongated structures (J. van de Koppel and C. M. Crain, personal observations).

To further investigate potential differences between models 2 and 3, we compared the large-scale qualitative properties of both models, using the bifurcation analysis program CONTENT (Kuznetsov and Levitin 1997), and we investigated the occurrence of spatial patterns over an extensive range of parameter values (see app. C for details). In our wetland system, senescence rate $s$, reflecting variation in mortality, may vary due to inundation time or herbivory by rodents. When senescence rate $s$ is varied, the analysis reveals important differences between the models. First, the range of $s$ along which spatial patterning is predicted (indicated by the blue and red lines in fig. 6) is much larger for model 3, the inhibition-at-intermediatedistance model (note the difference in the scale of the $s$ axis). Hence, patterning is a more robust prediction in model 3 than in model 2. Second, model 2 exhibits bistable dynamics along the entire range where patterns are predicted (as indicated by the dotted threshold line); both a patterned C. stricta vegetation state and a bare state without vegetation are stable. This has important implications for the potential development of hummocked vegetation. In model 3, plant establishment in a pristine environment, for instance by seed dispersal, would always lead to hummocked vegetation (as indicated by the arrow in fig. $6 B$ ). In model 2, however, a biomass threshold exists, preventing the establishment of vegetation on bare soil. This implies that in model 2, hummocked vegetation can develop from an existing homogeneous stand of C. stricta (e.g, at low $s$ ) in which conditions have deteriorated (e.g., due to increased inundation causing an increase in $s$ ), as indicated by the arrow in figure $6 \mathrm{~A}$. From this we can conclude that model 3 , with scale-dependent inhibition by wrack material, is a more plausible explanation for the observed regular spacing of C. stricta tussocks because homogeneous stands of $C$. stricta are rare in nature.

\section{Discussion}

In this study, we report on the presence and mechanisms driving regular tussock spacing in Carex stricta vegetation. Spectral analysis of aerial photographs indicated that tussock spacing was regular, with an average distance of about $60 \mathrm{~cm}$ between the tussocks. A transplantation experiment indicated that the strength of negative feedback from $C$. stricta by production of wrack material on aboveground growth of recruiting conspecifics varied with distance from the tussock, peaking at intermediate distances. Analysis of the relationship between availability of PAR and distance from the tussock revealed a strong decrease in PAR between 15 and $30 \mathrm{~cm}$ from the tussock, in agreement with the transplantation experiment. We then used mathematical models to investigate the applicability of three contrasting mechanistic explanations of the formation of regular pattern in marsh vegetation. The first mechanism is 

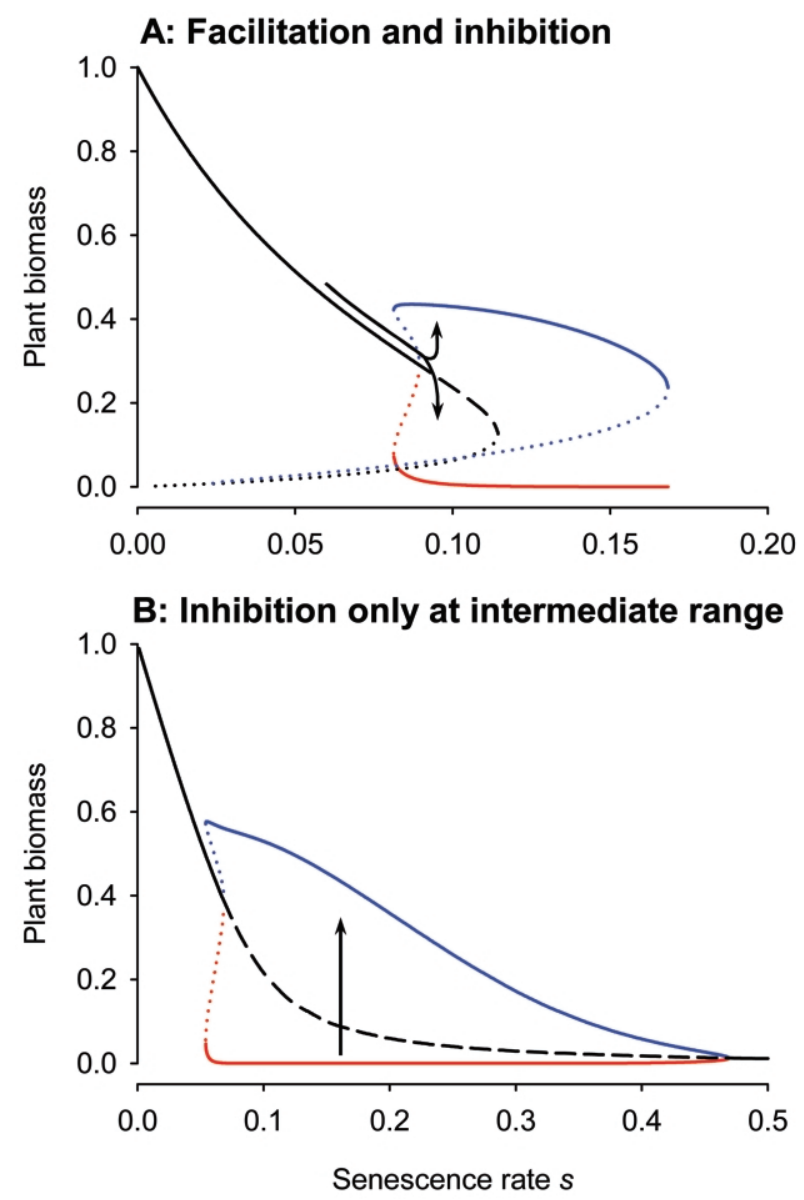

Figure 6: Bifurcation analysis for the facilitation-inhibition model ( $A$; model 2$)$ and the inhibition-at-intermediate-range model ( $B$; model 3). The graph presents the predicted biomass along a one-dimensionsal simulation domain of $0.4 \mathrm{~m}$ containing a single tussock as a function of the senescence rate $s$. The spatially homogeneous equilibria are in black, the maximal biomass within patterned vegetation (on top of the tussocks) is given in blue, and the minimal biomass (in the intertussock space) is in red. Dashed lines represent homogeneous equilibria that are unstable; patterns form when the equilibria are disturbed. Dotted lines represent threshold values, below which vegetation collapses to a bare state. The arrows depict possible routes for the development of patterned vegetation. Parameter values are similar to those in figure 5 except for $s$. Note the difference in the scale of the s-axis between graphs $A$ and $B$.

based on inhibition of neighboring individuals only and assumes a simple gradual decrease of intensity with increasing distance. The other two apply more complex scale-dependent principles. The second mechanism assumes an interaction between small-scale facilitation and large-scale inhibition. The third mechanism assumes that inhibition is strongest at intermediate distance and is alleviated locally, as was found in our experimental study. Our analysis revealed that the third model, in which $C$. stricta limits the growth of conspecifics through wrack production, but only at a distance from the tussock, explained patterning for an extensive range of parameter values. Moreover, while our experimental study investigates mechanisms driving tussock maintenance, the model ex- pands on this by indicating that only scale-dependent inhibition provides a putative explanation for the establishment of tussock vegetation on bare soil. Our combined experimental and mathematical results point at scaledependent inhibition of the growth of C. stricta by its own wrack as the most likely mechanism for the observed regularity in tussock spacing.

Our results contrast with earlier studies on the formation of regular spacing of individuals, as found in the literature concerning self-thinning within even-aged, single-species stands of plants (Ford 1975; Nisbet et al. 1997). Most of these studies point at intraspecific inhibition due to light competition as the cause of regularity in even-aged monocultures, with the (implicit) assump- 
tion that inhibition of neighbors by a specific individual decreases with distance from that individual (Kenkel 1988; Ford and Sorrensen 1992). Our empirical work, in combination with model analysis, emphasizes that inhibition decreasing with distance alone is an insufficient explanation for the observed regular spacing of Carex tussocks. Pattern generation requires inhibition to be weak locally, in order not to limit the individual's own growth, and to be strong at distance, limiting the growth of neighboring plants. This mechanism was highlighted by our experimental study, where tussocks elevated new shoots through the wrack canopy, ameliorating light reduction. In this respect, our results concur with studies in other systems such as arid bushlands (Klausmeier 1999; Von Hardenberg et al. 2001; Rietkerk et al. 2004b), peatlands (Rietkerk et al. 2004a), and mussel beds (van de Koppel et al. 2005) in stressing the importance of scale-dependence of feedback processes to explain regular patterning in ecosystems.

We were unable to generate regular spatial patterning in our model simulations when using the conventional decreasing relation between inhibition and distance from the originating individual. This raises the question of whether this mechanism acts in nature, as has been reported in examples of regular spacing in individual plants, or whether more complex elements have been overlooked. Direct or indirect facilitative effects of individuals on their own growth at local scale, which in turn drives spatial patterns in inhibition intensity, may have been unnoticed in these systems. A recent study on regular spacing in Arabidopsis thaliana, however, provides a key argument for this question (Stoll and Bergius 2005). It emphasized the importance of asymmetry in competitive interactions between individuals; regular patterns developed only when larger plants had a growth advantage that was more than proportional to their size. This can be interpreted as a facilitative effect of size on the growth of larger individuals, further enhancing size differences and causing sizedependant mortality. Hence, increased size results in a positive feedback for the involved organism but has a more than proportional negative effect on nearby competitors. Scale differentiation between local inhibitory effects of individuals on their own growth and inhibitory effects on neighboring plants seems key in both Stoll and Bergius (2005) and this study, suggesting a common principle that may apply to a broad range of regularly spaced stands of plants.

A number of studies suggest that tussock formation and the development of endogenous spatial structure within vegetation may have important consequences for community structure, particularly for species interactions and biodiversity. A recent study on the effects of tussock formation by Triglochin maritimum showed that a number of species were dependent on the indirect positive effects (reduced waterlogging and salinity) of T. maritimum tussocks (Fogel et al. 2004). In a prior study on C. stricta, positive associations were similarly found as tussocks protected other species against the adverse effects of herbivory by providing a spatial refuge from herbivores (Crain and Bertness 2005). Other species may benefit from the generation of spatial structure, since microhabitat heterogeneity has been shown to promote species diversity (VivianSmith 1997) and competition outside of aggregations can be reduced (Stoll and Prati 2001). While mathematical theory has extensively addressed the effects of spatial patterning on biodiversity (see Bolker et al. 2003 for an overview), the number of empirical studies addressing the effects of endogenous aggregation on species interactions is limited (Murrell et al. 2001). Empirical studies that investigate either the mechanisms behind endogenous patterning in ecosystems, as this study did, or the effects of endogenous patterning on species interactions (Stoll and Prati 2001; Fogel et al. 2004; Ervin 2005) will contribute significantly to our general understanding of the mechanisms driving biodiversity patterns in various ecosystems.

The model presented in the latter part of our study illustrates that the processes we documented can indeed explain the observed regular patterning. The model falls into the general category of Turing-type models, which explain regular patterning in ecosystems (or any other system) by assuming a local activating and a large-scale inhibiting process (Turing 1952; see Meinhardt 1995 for an intuitive explanation). Although this model has been hypothesized to explain regular patterning in a wide range of ecosystems, our study provides the first experimental evidence that this principle explains regular spatial patterning in an ecological system. Thereby, our research provides important evidence for the applicability of Turing's principle to the spatial complexity in ecological systems.

\section{Acknowledgments}

We thank A. Altieri, M. Bertness, M. Rietkerk, B. Silliman, and B. van Wesenbeeck for constructive comments on earlier drafts of this manuscript. The research was funded by a grant from the Schure-Beijerinck-Popping Fund to J.v.d.K. and an NOAA National Estuarine Research Reserve System Graduate Research Fellowship to C.M.C. This is publication 3915 of the Netherlands Institute of Ecology.

\section{APPENDIX A}

\section{Matlab Code for the Spectral Analysis}

The Matlab code that we developed for the spectral analyses in the article (see fig. 2 for output) is provided as a zip-compressed file (2D_Spectral_Analysis.zip), together 
with the two images on which the analysis in figure 2 is based and an explanatory text file. The code has not been peer-reviewed, and neither the journal nor the authors are able to provide support.

\section{APPENDIX B}

\section{Detailed Description of the Models}

Here we give a more detailed description of the models used to compare the three competing mechanistic explanations of tussock spacing that were described in the main text. The models all used a logistical growth function $P(1-P)$, which assumes $P$ to range in between 0 and 1 , where at $P=1$, self-limitation (e.g., by self-shading) reduces growth to 0 . The models furthermore assume a specific rate of leaf senescence $s$ and that all senesced material becomes wrack. Wrack decomposes with a specific rate $b$. Spatial dispersal of vegetation (through tillers) and wrack (resulting from dead plant leaves dropping to the soil surface and movement by the tides) are modeled using a diffusion approximation, where the rate of dispersal of wrack $d_{\mathrm{w}}$ exceeds that of tillers $d_{\mathrm{p}}$.

The first model investigates the potential for pattern formation when only the inhibitory effects of wrack on plant growth are considered:

$$
\begin{aligned}
& \frac{d P}{d t}=P(1-P)-s P-i P W+d_{\mathrm{P}} \Delta P, \\
& \frac{d W}{d t}=s P-b W+d_{\mathrm{w}} \Delta W .
\end{aligned}
$$

Here, inhibition is modeled by the linear term $i P W$, where $i$ is an inhibition coefficient.

The second model considers the implications of facilitation of plant growth by the formation of a root mound, modeled as a positive effect of vegetation on its own growth:

$$
\begin{aligned}
\frac{d P}{d t} & =P(1-P) \frac{P}{P+k_{2}}-s P-i P W+d_{\mathrm{P}} \Delta P, \\
\frac{d W}{d t} & =s P-b W+d_{\mathrm{w}} \Delta W .
\end{aligned}
$$

Here the term $P /\left(P+k_{2}\right)$ models the increase of growth due to facilitation by the root mound, assuming that the size of the mound increases linearly with the amount of plant biomass. Hence, it assumes that without a root mound, growth is close to 0 and the per capita growth increases to 1 as plant growth increases. The quantity $k_{2}$ represents the plant biomass where the facilitation term is half maximal.

The third model assumes an indirect facilitation of growth by the root mound by lowering of inhibition by the wrack:

$$
\begin{aligned}
\frac{d P}{d t} & =P(1-P)-s P-i P W \frac{k_{3}}{P+k_{3}}+d_{\mathrm{P}} \Delta P, \\
\frac{d W}{d t} & =s P-b W+d_{\mathrm{W}} \Delta W .
\end{aligned}
$$

Here $k_{3} /\left(P+k_{3}\right)$ is added to the inhibition term, lowering inhibition as $P$ increases. In this equation, $k_{3}$ represents the amount of plant biomass where inhibition is half maximal.

\section{Matlab Simulations}

Pattern development in the models is analyzed by simulating the spatial structure of the vegetation on a spatial grid of $512 \times 512$ cells with periodic boundary conditions, reflecting an area of $5 \mathrm{~m} \times 5 \mathrm{~m}$. At the start of the simulation, we seeded the area by increasing biomass in 200 randomly chosen cells to 1 while the other cells remained at 0 (note that we used such a high initial density to avoid the threshold effects for model 2). Simulations were run for 2,000 time steps, in abstract units, because our basic description of plant growth is nondimensional. For parameter values, see simulation results below.

The MatLab code we used to simulate the three models is in a zip archive: TussockModels.m. Note that there is a single code that simulates all models; the model number is set in line 6 . The code has not been peer-reviewed, and neither the journal nor the authors are able to provide support.

\section{APPENDIX C}

\section{Bifurcation Analysis with CONTENT}

The sensitivity of the model to changes in senescence rate $s$ with regard to pattern formation was analyzed with the help of CONTENT, a software program for numerical bifurcation analysis of dynamical systems (Kuznetsov and Levitin 1997, http://www.math.uu.nl/people/kuznet/ CONTENT/; Kuznetsov 1998). In CONTENT, a onedimensional version of the model with a spatial range containing a single peak (e.g, tussock) is analyzed. The program first establishes the equilibrium for a particular value of the bifurcation variable ( $s$ in this case) and then numerically traces this equilibrium using a continuation algorithm, identifying bifurcation points where the qual- 
itative properties of the equilibrium change (e.g., by becoming unstable). The results of this analysis are described in the main text.

\section{Literature Cited}

Bolker, B. M., S. W. Pacala, and C. Neuhauser. 2003. Spatial dynamics in model plant communities: what do we really know? American Naturalist 162:135-148.

Costello, D. 1936. Tussock meadows in southeastern Wisconsin. Botanical Gazette 97:610-648.

Couteron, P., and O. Lejeune. 2001. Periodic spotted patterns in semiarid vegetation explained by a propagation-inhibition model. Journal of Ecology 89:616-628.

Crain, C. M., and N. D. Bertness. 2005. Community impacts of a tussock sedge: is ecosystem engineering important in benign habitats? Ecology 86:2695-2704.

Ervin, G. N. 2005. Spatio-temporally variable effects of a dominant macrophyte on vascular plant neighbors. Wetlands 25:317-325.

Figueroa, M. E., J. M. Castillo, S. Redondo, T. Luque, E. M. Castellanos, F. J. Nieva, C. J. Luque, et al. 2003. Facilitated invasion by hybridization of Sarcocornia species in a salt-marsh succession. Journal of Ecology 91:616-626.

Fogel, B. N., C. M. Crain, and M. D. Bertness. 2004. Community level engineering effects of Triglochin maritima (seaside arrowgrass) in a salt marsh in northern New England, USA. Journal of Ecology 92:589-597.

Ford, E. D. 1975. Competition and stand structure in some evenaged plant monocultures. Journal of Ecology 63:311-333.

Ford, E. D., and K. A. Sorrensen. 1992. Theory and models of interplant competition as a spatial process: populations, communities, and ecosystems. Pages 363-406 in D. L. DeAngelis and L. J. Gross, eds. Individual-based models and approaches in ecology. Chapman \& Hall, New York.

Holmes, E. E., M. A. Lewis, J. E. Banks, and R. R. Veit. 1994. Partial differential equations in ecology: spatial interactions and population dynamics. Ecology 75:17-29.

Kenkel, N. C. 1988. Pattern of self-thinning in jack pine: testing the random mortality hypothesis. Ecology 69:1017-1024.

Klausmeier, C. A. 1999. Regular and irregular patterns in semiarid vegetation. Science 284:1826-1828.

Kuznetsov, Y. A. 1998. Elements of applied bifurcation theory. Springer, New York.

Kuznetsov, Y. A., and V. V. Levitin. 1997. CONTENT: a multiplatform environment for continuation and bifurcation analysis of dynamical systems (software under development). Centrum voor Wiskunde en Informatica, Amsterdam.
Lejeune, O., M. Tlidi, and P. Couteron. 2002. Localized vegetation patches: a self-organized response to resource scarcity. Physical Review E 66:010901.

Meinhardt, H. 1995. The algorithmic beauty of sea shells. Springer, Berlin.

Mugglestone, M. A., and E. Renshaw. 1998. Detection of geological lineations on aerial photographs using two-dimensional spectral analysis. Computers and Geosciences 24:771-784.

Murrell, D. J., D. W. Purves, and R. Law. 2001. Uniting pattern and process in plant ecology. Trends in Ecology \& Evolution 16:529530

Nisbet, R. M., S. Diehl, W. G. Wilson, S. D. Cooper, D. D. Donaldson, and K. Kratz. 1997. Primary-productivity gradients and short-term population dynamics in open systems. Ecological Monographs 67: 535-553.

Powell, J. A., and N. E. Zimmermann. 2004. Multiscale analysis of active seed dispersal contributes to resolving Reid's paradox. Ecology 85:490-506.

Renshaw, E., and E. D. Ford. 1983. The interpretation of process from pattern using two-dimensional spectral analysis: methods and problems of interpretation. Applied Statistics 32:51-63.

Rietkerk, M., S. C. Dekker, M. J. Wassen, A. W. M. Verkroost, and M. F. P. Bierkens. 2004a. A putative mechanism for bog patterning. American Naturalist 163:699-708.

Rietkerk, M., S. C. Dekker, P. C. de Ruiter, and J. van de Koppel. 2004b. Self-organized patchiness and catastrophic shifts in ecosystems. Science 305:1926-1929.

Stoll, P., and E. Bergius. 2005. Pattern and process: competition causes regular spacing of individuals within plant populations. Journal of Ecology 93:395-403.

Stoll, P., and D. Prati. 2001. Intraspecific aggregation alters competitive interactions in experimental plant communities. Ecology 82: 319-327.

Turing, A. M. 1952. The chemical basis of morphogenesis. Philosophical Transactions of the Royal Society B: Biological Sciences 237:37-72.

van de Koppel, J., M. Rietkerk, N. Dankers, and P. M. J. Herman. 2005. Scale-dependent feedback and regular spatial patterns in young mussel beds. American Naturalist 165:E66-E77.

Vivian-Smith, G. 1997. Microtopographic heterogeneity and floristic diversity in experimental wetland communities. Journal of Ecology 85:71-82.

Von Hardenberg, J., E. Meron, M. Shachak, and Y. Zarmi. 2001. Diversity of vegetation patterns and desertification. Physical Review Letters 87:198101-198104.

Associate Editor and Editor: Donald L. DeAngelis 